\title{
CCD Astrometry and Photometry of Visual Double Stars: Northern Hipparcos Wide Pairs Measured in the Years 2003-2005
}

\author{
D. Sinachopoulos ${ }^{1}$, P. Gavras ${ }^{1,2}$, Th. Medupe ${ }^{3}$, \\ Ch. Ducourant ${ }^{4}$, and O. Dionatos ${ }^{2}$ \\ ${ }^{1}$ National Observatory of Athens, Institute of Astronomy and Astrophysics \\ ${ }^{2}$ National and Kapodistrian University of Athens, \\ Department of Astronomy, Astrophysics and Mechanics \\ ${ }^{3}$ South African Astronomical Observatory \\ ${ }^{4}$ Observatory of the University of Bordeaux
}

\begin{abstract}
The relative positions of Hipparcos visual double star components are currently known with a precision around fifty mas. Modern CCD astrometric observations of these objects achieve an accuracy of their angular separation between ten and twenty mas per observation. New CCD measurements have been obtained at Kryonerion Observatory in the north hemisphere. They provide current relative positions of visual double stars which are at least twice as accurate as the ones provided by Hipparcos. The new measurements will permit us to extract the physical pairs from the sample, and the double stars, which have components of common origin. Final statistics of these systems will improve our understanding of stellar formation and evolution rates of wide binaries in the solar neighborhood.
\end{abstract}

Keywords. stars: wide double stars: Hipparcos - double stars- visual - astrometry - differential photometry

\section{Introduction}

The data in the Hipparcos catalogue refer to mean epoch 1991.25 (Perryman et al. 1997). The proper motions of the catalogue were based on the three years of the satellite observations and according to Lindegren et al. (1997). they have a mean precision of 25 mas per year. This value is valid mainly for the single stars, since the precision is lower for systems with angular separations in the range 2 to 12 arcseconds (Oblak et al. 1999). Therefore now, more than one decade after the Hipparcos catalogue epoch, the relative positions of the Hipparcos visual double star components listed in the Hippacos catalogue are known with a precision worse than 50 mas.

Contemporary astrometric CCD observations of such objects usually achieve an angular separation accuracy of about 15 mas per observation, as it is shown by Sinachopoulos et al. (1988). This is at least a factor of three higher than the precision of the Hipparcos positions now.

For a detailed statistical study of the Hipparcos wide double stars is necessary to obtain new CCD measurements. We performed these observations during the last two years using the 1.2-m telescope of the Kryonerion Astronomical Station of the National Observatory of Athens.

It is our intention to use our new measurements together with the Hipparcos data and all old observations known, in order to determine the most accurate relative proper 
motion of the components of these double stars published since Brosche \& Sinachopoulos (1988, 1990). These precise relative proper motions will permit the extraction of the physical pairs and the double stars which have components of common origin from the double stars of the sample. This will allow the estimation of the rate of occurrence of wide double stars in the solar neighbourhood.

We present here results for 221 Hipparcos northern visual double stars. In 63 of the cases, both components are Hipparcos stars and for other observed double stars only one component has an entry in the Hipparcos catalogue.

Our new results are also a continuation of the observing program initiated in 1988 (Sinachopoulos et al. 1988) . Further major contributions in the field are published by Argue et al. (1992) and Oblak et al. (1999).

\section{Observations}

The 1.2-m Kryonerion telescope has been used for a period of 2.5 years starting in the summer of 2003. Scheduled observations have been performed according to weather conditions and telescope availability. Details on the $1.2-\mathrm{m}$ telescope and the performance of its CCD camera have been described by Sinachopoulos et al. (1999).

The photometric quality nights were mainly concentrated in the summer months. Unfortunately, weather and telescope conditions do not allow all-sky photometry, so we were not able to transform our photometric data onto the standard photometric $U B V R I$ system. Nevertheless, accurate magnitude difference of the double star components could be calculated due to the very small angular separation of the two stars.

Average seeing was $1^{\prime \prime} .8$ and no systematic seasonal seeing variations could be determined. This seeing is mainly caused by the telescope dome, since some simultaneous measurements made outside the telescope building using the DIMM method found seeing values around one arcsecond.

Sixteen exposures per visual double star have been done usually in the Bessel $V$ filter. Thirty percent of the targets were observed in the Bessel $I$ filter as well in order to search for close low mass companions.

\section{Data reduction}

The CCD camera linearity was found to go up to 30000 ADUs remaining always better than $1 \%$. Cosmic ray hits, bias subtraction and flat-field division was performed to the observations using tools of the IDL software package.

A small percentage of the exposures could not be reduced due to tracking errors of the telescope.

Most of the double stars in our sample are very wide pairs and the images of their components do not overlap. In these cases, two separate Gaussians were fitted to the images and corresponding aperture photometry was used for deriving the components magnitude difference. We developed a new program in IDL for this part of the data reduction. For the overlapping components we used our FORTRAN program, discussed in Sinachopoulos et al. (1999). This fits simultaneously two MOFFAT profiles to both components. A detailed description of the MOFFAT profile can also be found in Shatsky et al. (1999).

\subsection{Calibration of the position angle}

Stellar traces near the equator and meridian have been taken every night in order to determine the deviation (offset) of the CCD camera orientation from the East-West 

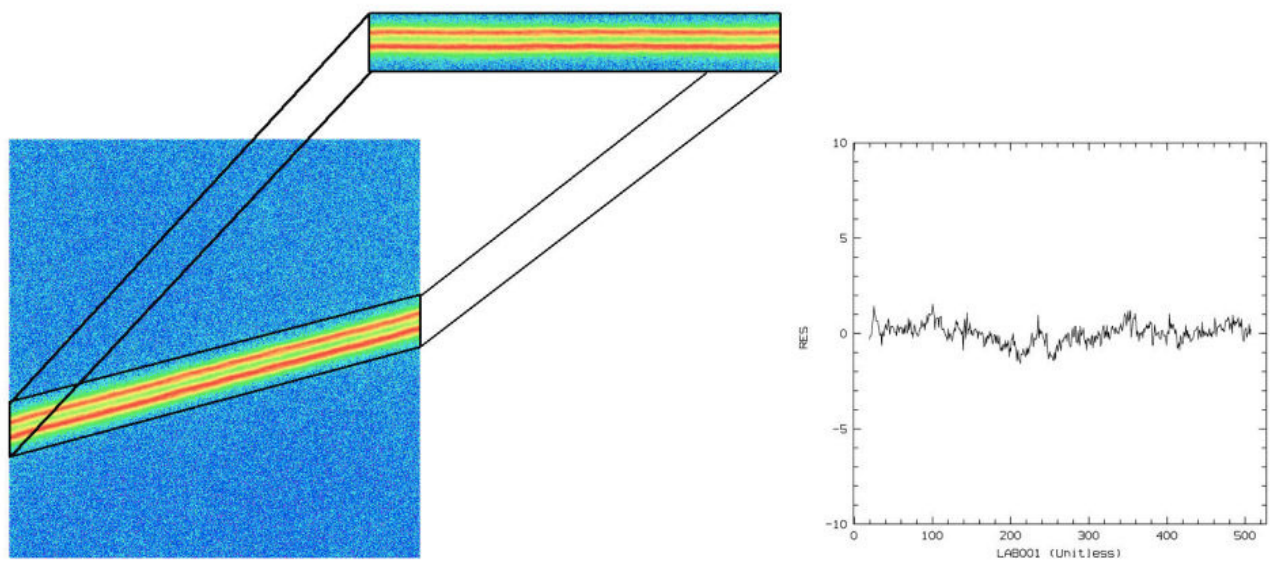

Figure 1. Stellar traces and $O-C$ photocenter positions in arcseconds, taken with seeing $2^{\prime \prime} .3$.

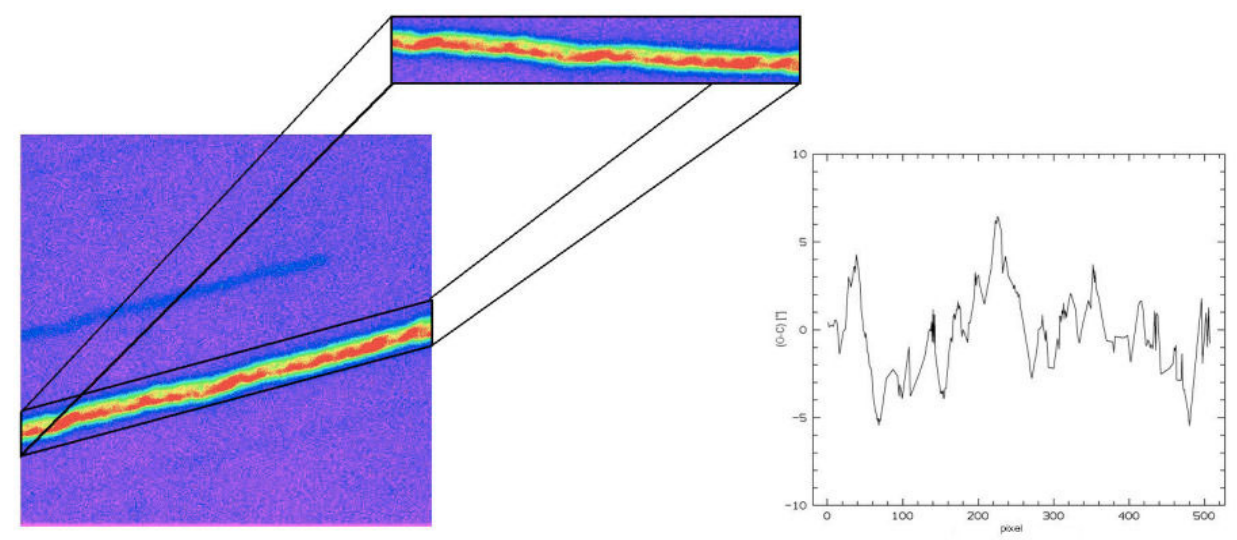

Figure 2. Stellar traces and $O-C$ photocenter positions in arcseconds, taken under extremely poor seeing

direction. This correction has been applied to the instrumental position angle measured on the CCD in order to determine the direction of the North.

ESO-MIDAS software has been used for the reduction of these observations. Our algorithm first detects all stellar traces on each CCD frame. Each one of the detected stellar traces was treated separately. We determine first the photocenter position of the trace on each of the CCD frame columns by fitting a one dimensional Gaussian. We determine in this way the position of the trace in columns of the frame. Finally, a linear regression is performed to the measured positions of the stellar trace and we determine in this way the inclination of the stellar trace in respect to the CCD detector. This inclination shows the correct East-West direction on the CCD chip.

The differences of the photocenter position determinations between the observed trace and the positions determined by the linear regression fitting characterize the quality of the observing conditions. Figure 1 shows such differences during seeing of about $2^{\prime \prime} .3$ and Figure 2 during one of the worst seeing conditions measured at this telescope. 
Table 1. Dependency of the inclination of star trails on declination, as well as the slope of the inclination inside HA $\cos (\delta)$ arcs studied in each declination interval. Values are given in degrees.

\begin{tabular}{|c|c|c|c|c|c|c|c|c|}
\hline \multicolumn{4}{|c|}{ Validity range } & \multirow{2}{*}{\multicolumn{2}{|c|}{$\begin{array}{l}\text { Inclination } \\
\text { of trails }(i)\end{array}$}} & \multirow{2}{*}{\multicolumn{2}{|c|}{$\begin{array}{c}\text { Slope of } \\
\text { inclination }\left(S_{i}\right)\end{array}$}} & \multirow{2}{*}{$\begin{array}{l}\text { No of } \\
\text { trails } \\
\text { used }\end{array}$} \\
\hline $\begin{array}{c}\text { Decl } \\
\left({ }^{\circ}\right)\end{array}$ & $\begin{array}{c}\text { iation } \\
\left({ }^{\circ}\right)\end{array}$ & $\begin{array}{l}\mathrm{HA} \\
\left({ }^{\circ}\right)\end{array}$ & $\begin{array}{r}\mathrm{OS}(\delta) \\
\left(\left(^{\circ}\right)\right.\end{array}$ & & & & & \\
\hline-05 & +35 & -45 & +45 & +0 . & 0.004 & -0 . & 0 & 597 \\
\hline+36 & +50 & -15 & +40 & +0.008 & 0.045 & -0.0066 & 0.0022 & 88 \\
\hline+51 & +65 & -10 & +30 & +0.140 & 0.043 & -0.0065 & 0.0023 & 75 \\
\hline+66 & +78 & -04 & +05 & +0.17 & 0.14 & & & 24 \\
\hline+83 & +85 & -02 & +03 & +0.58 & 0.10 & & & 8 \\
\hline
\end{tabular}

We performed one more study in order to determine the additional corrections which have to be applied to the measured position angles due to the misalignment between the axis of the rotation of the telescope and the axis of the Earth. More than eight hundred stellar traces have been taken for this purpose.

During these exposures the telescope was pointing to selected zones of declination and hour angles (HA). Table 1 shows the systematic inclination differences of the stellar traces observed at different hour angles and declinations. In this table, the trace inclination were set to zero for the telescope pointing to the equator and the meridian.

We studied the dependency of the inclination of star trails (in degrees), as well as the slope of the inclination inside different intervals of $\mathrm{HA} \cos (\delta)$ and declination.

About 600 such star trails were taken in declinations between $-5^{\circ}$ and $+35^{\circ}$ and an hour angle range equivalent to an interval of $-45^{\circ}$ to $+45^{\circ}$ from the meridian calculated as $\mathrm{HA} \cos (\delta)$. All observations taken in this part of the sky show parallel stellar traces at an accuracy level of $0^{\circ} .004$. Over the declination range $+36^{\circ}$ to $+65^{\circ}$ we found that corrections should be applied to the position angle measurement according to the formula

$$
\Delta \theta=i+S_{i} \cdot H A \cos (\delta)
$$

using the corresponding values of Table 1 . For declinations between $+66^{\circ}$ and $+85^{\circ}$ the inclination values of the traces determined - as well as the inclination slope computed for different $\mathrm{HA} \cos (\delta)$ values — are valid for observations around the meridian only. Since there are only a relatively small number of double stars at declinations north of $+66^{\circ}$ we took star trails each time we observed a double star there.

Column three of Table 1 shows the measured inclination of the trace and its accuracy in degrees for the declination zone listed in column one and hour angle (multiplied by $\cos (\delta)$ ) inside the declination zone listed in column two. Column four contains the slope of the inclination and its accuracy in column three. In the last column is listed the number of CCD frames used for obtaining the values of column three and four.

\subsection{Calibration of the angular separation}

The scale of the 1.2-m telescope was first determined by Sinachopoulos et al. (1999) and found to be $0.30118 \pm 0.00056$ arcseconds/pixel (the pixel size of this CCD camera is $24 \times 24$ microns) by using ten Hipparcos stars which were components of five visual double stars. The CCD camera is the same, but we now used the Hipparcos catalogue data for both components of 63 double stars in order to improve upon the previous value. The new scale estimation is $0.30033 \pm 0.00015$ arcseconds/pixel. Figure 3 shows the distribution of the telescope scale values estimated for the 63 Hipparcos double stars used. The old and new scale values are statistically the same to a very high level of significance. 


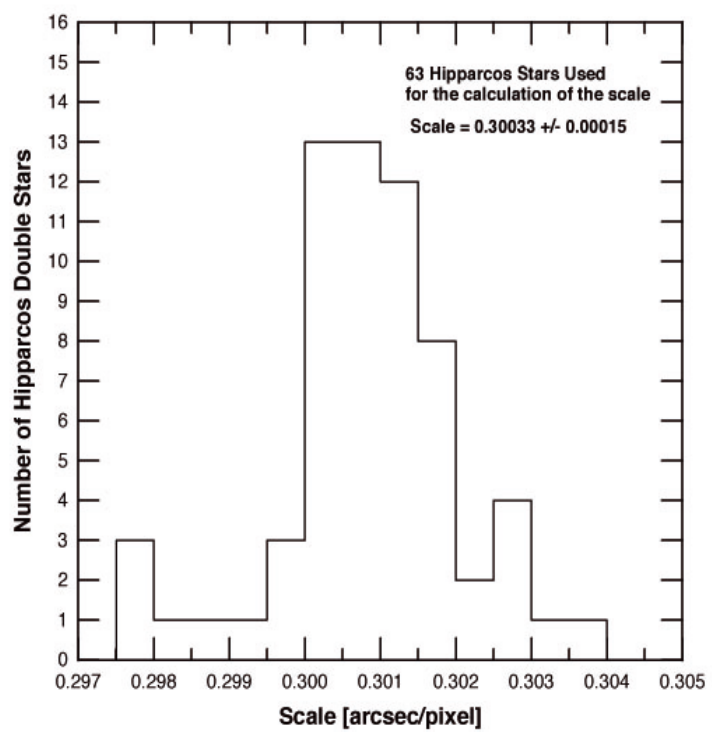

Figure 3. Distribution of the telescope scale determination using Hipparcos double stars

Table 2. Astrometric results and component magnitude differences in the Bessel $V$ filter

\begin{tabular}{|c|c|c|c|c|c|c|c|c|c|}
\hline $\begin{array}{l}\alpha_{2000} \\
\mathrm{~h} \quad \mathrm{~m} \quad \mathrm{~s}\end{array}$ & $\circ \delta_{2000}^{\prime \prime}$ & WDS & $\rho$ & $\sigma_{\rho}$ & $\theta$ & $\sigma_{\theta}$ & $\begin{array}{c}\Delta V \quad \sigma_{\Delta V} \\
\text { magnitudes }\end{array}$ & $\begin{array}{l}\text { Epoch } \\
\text { year }\end{array}$ & No \\
\hline $\begin{array}{lll}1 & 52 & 10.1\end{array}$ & +662656 & $01522+6627$ & 29.515 & 0.016 & 316.20 & 0.02 & $2.695 \quad 0.003$ & 2003.60 & 15 \\
\hline $\begin{array}{lll}2 & 42 & 21.2\end{array}$ & $+38 \quad 3708$ & $02424+3837$ & 14.562 & 0.008 & 343.40 & 0.01 & $\begin{array}{ll}1.521 & 0.002\end{array}$ & 2004.91 & 16 \\
\hline $\begin{array}{llll}2 & 55 & 47\end{array}$ & $+34 \quad 2840$ & $02558+3429$ & 21.519 & 0.012 & 148.77 & 0.01 & 1.4210 .001 & 2004.91 & 16 \\
\hline $\begin{array}{lll}3 & 02 & 16.8\end{array}$ & +412345 & $03023+4124$ & 17.602 & 0.010 & 162.86 & 0.01 & 1.8220 .002 & 2004.91 & 16 \\
\hline
\end{tabular}

\section{Astrometric and photometric results}

We obtained new astrometric measurements and magnitude differences of the components of 211 northern wide visual double stars. Table 2 shows a small portion of these results (The final catalogue will be available at CDS). The first two columns contain the 2000 coordinates of the double star and the third column its WDS identifier. Column four shows the component angular separation and its accuracy in arcseconds. Column five contains the position angle of the double star components and its accuracy (in degrees), and column six the corresponding magnitude difference in $V$ and its accuracy. Column seven shows the epoch of the observation and last column the number of the exposures used during the data reduction.

In addition, sixty double stars of the sample have been observed in the Bessel $I$ filter as well. The astrometric results are practically the same in both filters. An additional table has been created for the $I$ observations, containing the WDS identifier, the component magnitude differences in $I$ with the achieved accuracy, the observation epoch and the number of exposures used. 


\section{Conclusions}

The detailed study of the $1.2-\mathrm{m}$ telescope axis misalignment shows that additional corrections of the position angle should be taken when double stars are observed in declinations northern to $35^{\circ}$.

On the other hand, the telescope scale determined here using 63 Hipparcos double stars should be considered a final one.

Similarly to previous papers of CCD astrometry, the final accuracy level of the present results is the same with the one of the Hipparcos mission for systems with angular separations in the range 2 to 10 arcseconds.

Any further interpretation, such as an in-depth determination of the physical status of components, exceeds the scope of this poster.

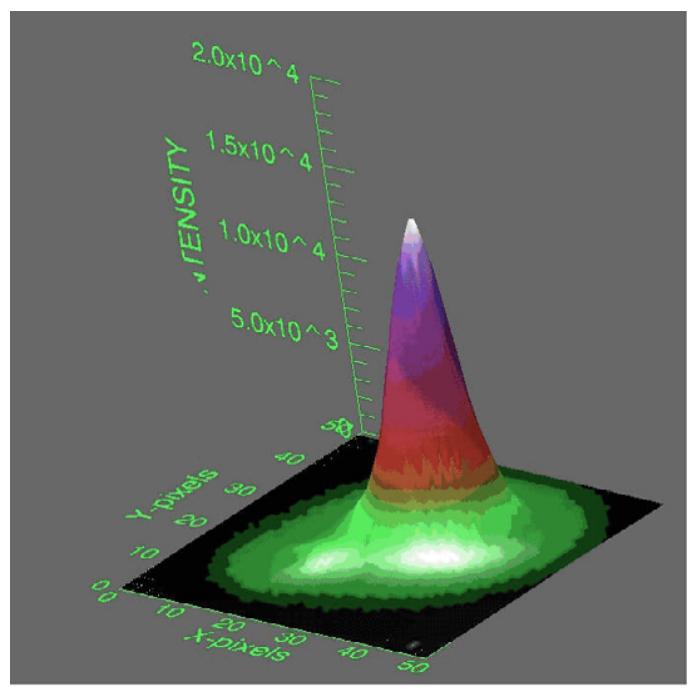

Figure 4. The double star WDS $12016+7051$ as fitted by our IDL procedures

\section{Acknowledgements}

This research was partially carried out in the framework of the project ATHENOGAIA, financed by the Greek General Secretary for Research and Development.

D. Sinachopoulos would like to thank the Universities of Bordeaux (France) and SouthWest (South Africa) for support and accommodation.

\section{References}

Argue, A.N. et al. 1992, MNRAS. 259, 563

Brosche, P. \& Sinachopoulos D. 1988, BICDS 34, 39

Brosche, P. \& Sinachopoulos D. 1990, BICDS 37, 109

Lindegren, L. et al. 1997, A\&A A323, L53-L56

Oblak, E. et al. 1999, A\&̈A. 346, 523

Perryman, M. et al. 1997, A\&\&A 323, L49-L52

Shatsky, N., Sinachopoulos, D., Prado. P., \& van Dessel, E. 1999, A\&AS. 139, 69

Sinachopoulos D., Geffert, M., \& Nicklas, H. 1988, Ap\&SS 142, 227

Sinachopoulos D., Dapergolas, A., van Dessel, E., \& Kontizas, E. 1999, A $\& A S$ 136, 525 